\title{
Probing the Molecular Basis for the Lateral Flexibility of Tight Junction Strands
}

Evan S. Krystofiak ${ }^{1}$, Jun Zhao ${ }^{1}$, Angela Ballesteros Morcillo ${ }^{1}$, Christina M. Van Itallie ${ }^{3}$, Runjia Cui ${ }^{1}$, James M. Anderson ${ }^{3}$, Cristina Fenollar Ferrer ${ }^{2}$, and Bechara Kachar ${ }^{1}$

1. Laboratory of Cell Structure and Dynamics, National Institute on Deafness and Other Communication Disorders, Bethesda, MD US

2. Computational Structural Biology Unit, National Institute of Neurological Disorders and Stroke, Bethesda, MD US

3. Laboratory of Tight Junction Structure and Function, National Heart, Lung, and Blood Institute, Bethesda, MD US

Tight junctions (TJ) form selective ion permeable barriers in epithelial and endothelial tissues that are essential for many physiological processes. These barriers consist of a network of sealing strands between adjacent cells primarily composed of linear claudin oligomers. Strand formation requires coordinated claudin interactions within the same membrane (cis) and interactions between monomers from adjoining cells (trans). The recently reported x-ray crystal structure of claudin-15 [1] has provided a structural basis for modeling TJ strands, leading to a proposal of a linear multimeric strand model [2] largely based on a cis interface (X-1) observed in the crystal packing (Fig.1A). Claudin strands are dynamic and able to maintain their barrier function during cell movements and large scale tissue rearrangements. Here we examined lateral bending and flexibility of claudin strands and we propose an alternative cis interaction.

TJ strand networks and individual strands were examined by live imaging of cultured cells expressing GFP-tagged claudins. Individual claudin strands were very dynamic displaying isotropic lateral bending (Fig.1B). In order to examine the curvature of claudin strands beyond the resolution limits of confocal microscopy, we performed freeze fracture on cells expressing claudin-15 (Fig.1C) and determined the curvature distribution (Fig.1D). The strands primarily exhibited continuous curvature with a median curvature of $7.9 \mu^{-1}$, equivalent to bending of $1.3^{\circ}$ per monomer step. However, we observed curvatures of up to $122 \mu^{-1}\left(20^{\circ}\right.$ per monomer step) at strand inflection points. While the continuous low curvatures are consistent with the X-1 interface, the abrupt inflection points within the strands suggested that there may be alternative cis interfaces that support differing angles between protomers.

Computational protein-protein docking was employed to search for additional cis interfaces. We identified an alternative cis interface, named Cis-1, between conserved residues in the extracellular helix domain and the second extra cellular loop; it is similar to $\mathrm{X}-1$ but with a $16^{\circ}$ rotation between protomers (Fig.1E). When compared to X-1, Cis-1 exhibits more hydrogen bonding, a greater interface area, and more favorable interface energy, while both interfaces share intermolecular hydrogen bonds made by residues S67 and E157 (Fig.1F). Freeze fracture of S67 or E157 mutants demonstrated a distinct beaded strand morphology with proteins segregated to both the P- and E-faces (Fig.1G), compared to the continuous P-face strands observed in wild type claudin-15. The change in strand morphology supports that $\mathrm{S} 67$ and E157 residues are involved in normal claudin oligomerization. We argue that X-1 and Cis1 interfaces function as a hinge around hydrogen bonds that permits structural variability and strand flexibility. The X-1 and Cis-1 interfaces may be different states of this hinge region with the X-1 interface representing low curvature regions and Cis-1 the higher curvatures of the strand [3]. 


\section{References}

[1] Suzuki H et al, Science 344 (2014), p. 304.

[2] Suzuki H et al, Journal of Molecular Biology 427 (2015), p. 291.

[3] Supported by the National Institute on Deafness and Other Communication Disorders Intramural Research Program (Z01 DC000002) and the NIDCD Advanced Imaging Core (ZIC DC000081)
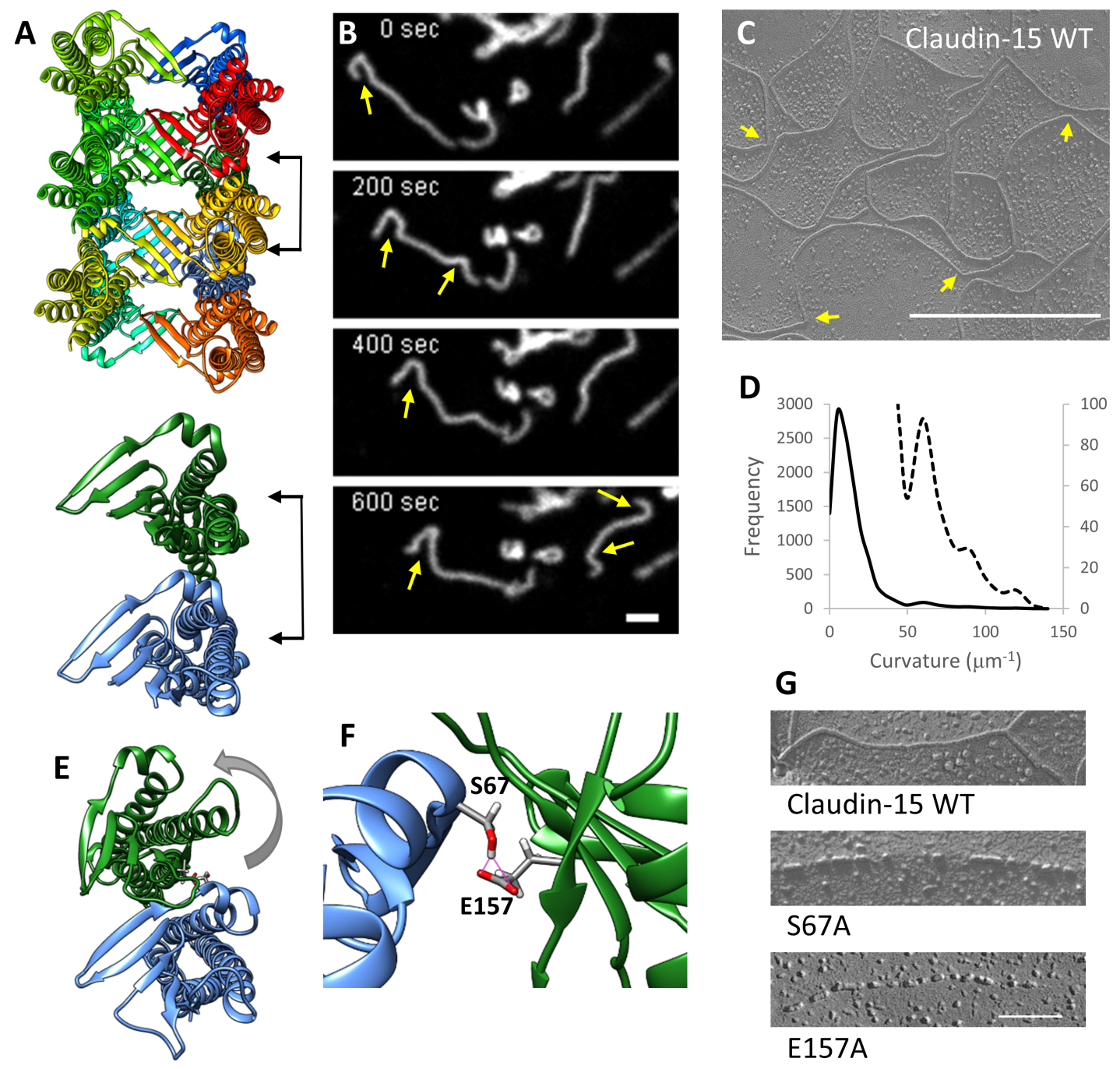

Figure 1. Claudin strand curvature and a hinge-like flexibility mechanism. (A) Double strand model based on Suzuki et al. [1] using the cis interface derived from x-ray crystal contacts, arrows indicate a single X-1 cis dimer. (B) Live cell imaging of tight junction strands formed by GFP-tagged claudin-2, arrows indicate high curvature regions. (C) Freeze fracture of wild type claudin-15 strands, arrows indicate strand inflections. (D) The curvature distribution of claudin-15 strands (solid) and the same distribution at a reduced frequency scale on the right, highlighting the high curvature distribution (dashed). (E) The Cis-1 interface derived from protein-protein docking contains hydrogen bonds between residues S67 and E157 (F). (G) Freeze fracture of claudin-15 mutants S67A and E157A show discontinuous strand morphologies with segregation to both the P- and E-faces; wild type claudin-15 strand are continuous and segregate to the P-face. Size bars are $1 \mu \mathrm{m}$ for (B), $500 \mathrm{~nm}$ for (C) and $100 \mathrm{~nm}$ for $(\mathrm{G})$. 